\title{
Revitalization of Fangyan Through Social Media Promotion in China
}

\author{
Haiyan Guo ${ }^{1}$, Zhijuan $\mathrm{Ni}^{1}$, Zhiying Wang ${ }^{1}$, Yu Zhang ${ }^{1}, \& \mathrm{Jia} \mathrm{Li}^{1}$ \\ ${ }^{1}$ School of Foreign Language, Yunnan University, Kunming, People's Republic of China \\ Correspondence: Zhijuan Ni, School of Foreign Language, Yunnan University, Kunming, People's Republic of \\ China. Tel: 135-1740-4701. E-mail: 1325002184@qq.com
}

Received: November 20, 2021

Accepted: January 16, 2022

Online Published: February 28, 2022

doi:10.5539/ass.v18n3p1

URL: https://doi.org/10.5539/ass.v18n3p1

\begin{abstract}
Compared to Putonghua, Fangyan is often indexed with social stereotypes such as lack of education, uncivilization and low-class. However, such negative indexicality of speaking Fangyan has been challenged by the emerging circulation of diverse social media online. Adopting the concept of language as commodity in late capitalism (Heller, 2010), this study examines how Fangyan is constructed and promoted as an index of authenticity and authority, a source of knowledge dissemination and commodified capital. The study argues that the revitalization of Fangyan from below cannot be simply reduced to the celebratory discourse of cultural diversity but should be understood in a wider discourse of language as profit which is subordinated to the power and social relations. The study can shed lights on the promotion of linguistic diversity and intercultural communication.
\end{abstract}

Keywords: Fangyan, social media, language as commodity, China

\section{Introduction}

The traditional conceptualization of speaking Fangyan are considered as "low prestige" or carrying some negative meanings. In the process of social urbanization, Fangyan provides identity and living space for the lower-class migrant workers (Fang, 2015). Dong found that people think that speaking in Fangyan is "unpleasant" and "funny", and Fangyans are even abnormalized (Dong, 2014). However, such negative indexicality of speaking Fangyan has been challenged by the new configuration of speaking Fangyan as resource/commodity. During the Covid-19 pandemic, Fangyan is functioned as an emergency language for people to gain health information $(\mathrm{Li}, 2020)$. In addition, the ethnic minority languages and their cultural products increasingly come to be seen as a form of capital to boost the local economy ( $\mathrm{Li}, 2020)$. Given the visibility and audibility of Fangyan has been observed, the ways to protect and revitalize it should be concerned, and one of which is to make a combination of social media and Fangyan (Cao, 2017; Spitulnik, 1999). Furthermore, there is still lack of empirical studies on how Fangyan is capitalized through the social media promotion in China, and lack of a holistic and comprehensive view of the ways how Fangyan and Putonghua are represented. Focusing on the participants' language practice in the "1.3 Billion Decibel" show, this paper offers a nuanced account of the commodification of Fangyan, and the tensions of languages under the Putonghua promotion policy. By so doing, the study hopes to shed light on the complex roles for Fangyan, and to contribute to the language protection and linguistic diversity globally.

\section{Research Context}

\subsection{The Dilemma of Fangyan}

Fangyans are facing a lot of challenges. On the one hand, Fangyans' linguistic rights are partial. China has about 135 languages in the five major language families of Sino-Tibetan, Altai, Austronesian, South Asian, and Indo-European (Cao, 2017). China is rich in language resources (see Figure 1). However, as the language policy from the national level is "Putonghua promotion", Putonghua, with its status surpassing other languages, become the only legitimate language to get education, employment and medical service. On the other hand, the rapid loss of Fangyan is becoming more and more serious. Among the more than 130 languages that exist in China, 68 languages speak a population of less than 10,000, of which 25 languages speak a population of less than 1,000, and some of them are minority languages with a population of less than 100 (Tian, 2015). Under the pressure of the two main dilemmas, the language situation faced by Fangyan is very severe. In order to protect Fangyan, starting from the national level, language protection projects have been started one after another. 


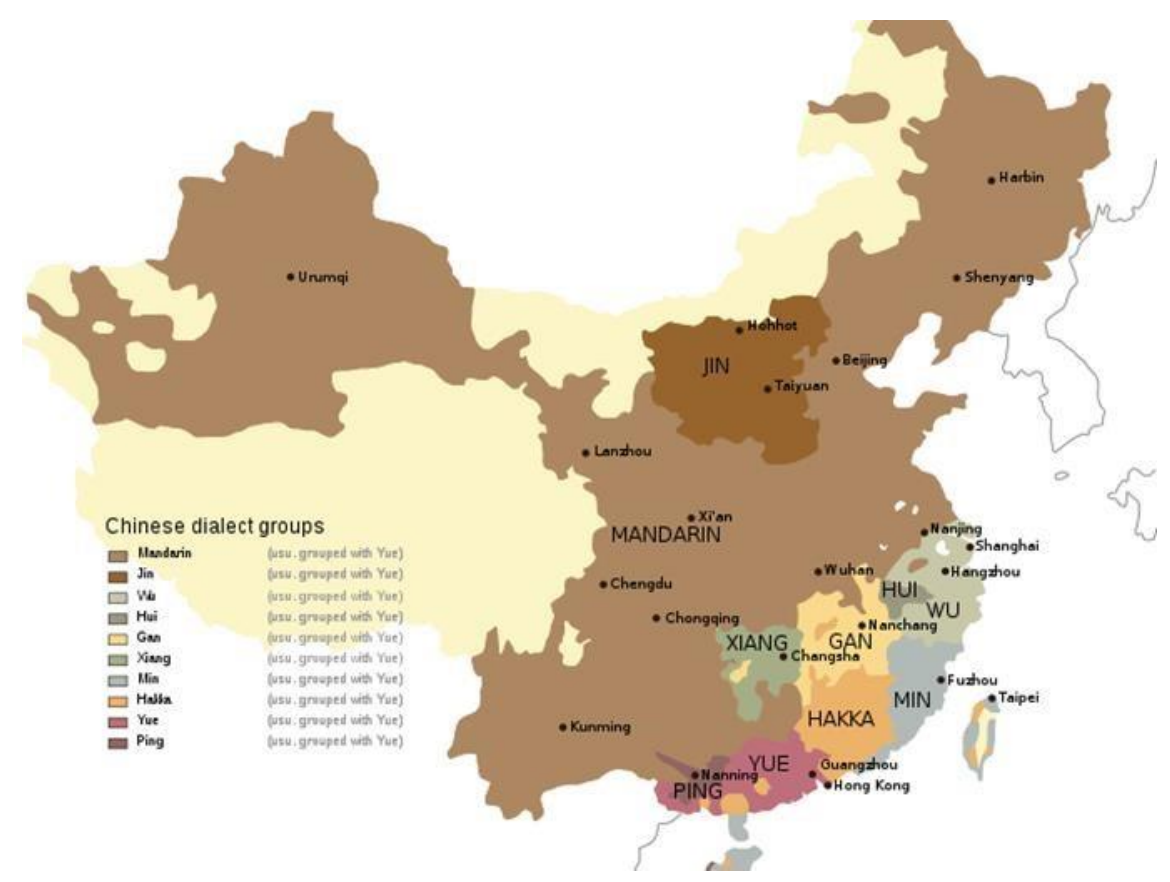

Figure 1. Language Atlas of China from Wikipedia

\subsection{The Increasing Popularity of Social Media}

Before the emergence of social media, the interpenetration of local Fangyan was always accompanied by the flow of people, and it was presented by interpersonal communication as the main communication method. Subsequently, the social media enabled the large-scale diffusion of dialects in the region, disseminating dialect cultural programs to local citizens in a relatively standard mode. In the paper, the social media refers to the Internet. According to "The 47th China Statistical Report on Internet Development" released by China Internet Network Information Center (CNNIC), China has over 989,000,000 Internet users by the end of 2020, which means the Internet penetration rate in China has reached 70.4\% (Mo, 2021). With the increasing number of Internet users, social media, as one of the applications of Internet, plays an important role in daily life. Urla (1995) pointed out that "in the period of big data, the role of social media is prominent, mass media may also be used as a powerful tool of subversion."

Facing the language challenges, some researchers are thinking about ways to protect Fangyan, one of which is to make a combination of Fangyan and social media (Spitulnik, 1999). Spitulnik (1996) demonstrated how mass media, because of their extensive accessibility and scope, can serve as both reservoirs and reference points for the circulation of words, phrases, and discourse styles in popular culture. In the study, Fangyan's value in the "1.3 Billion Decibel" show is explored with the following questions: (1) How is Fangyan commodified through the social media promotion? (2) What are the tensions embedded in the process of Fangyan's capitalization?

\section{Theoretical Framework}

This paper is informed by the commodification of language as the theoretical framework. Monica Heller (2003, 2010) illustrates "how the phenomenon of commodification occurs in a number of key sites of globalization in which language emerges as a symbolic capital becoming a resource with an exchange value - tourism, marketing, translation, communications and performance art." Furthermore, Heller (2010) proposed 2 ways, often competing, to commodify languages: one is as a technical skill, it can be managed by Taylorism techniques invented for industrialization (Urciuoli, 2001; Cameron, 2005), the other is as a sign of authenticity (Coupland, 2003a). In the study, Fangyan is commodified by the new technical skill, social media. Besides, in the theory of the commodification of language, there are tensions between standardization and variability, which represents a gap, or troubled space of contradiction, between established nationalist (Heller, 2010). In the study, there still exist some gaps between the standardized language, Putonghua, and the language varieties, Fangyans. 


\section{Methodology}

\subsection{Object of the Study}

The data of the study is selected from the show "1.3 Billion Decibel", which is the first domestic "Fangyan + music" show in China. By 2018, 1.3 Billion Decibel had been streamed 300 million times, and nearly 3 million people had live-streamed it online ( $\mathrm{Gu}, 2018)$. Because it is the first show make a successful combination of Fangyan and social media and it gains a high attention from audience in China, the show appears to stand with potential to be proceeded with. What's more, there are 21 episodes (from 2016-7-8 to 2016-9-23) in the first quarters.

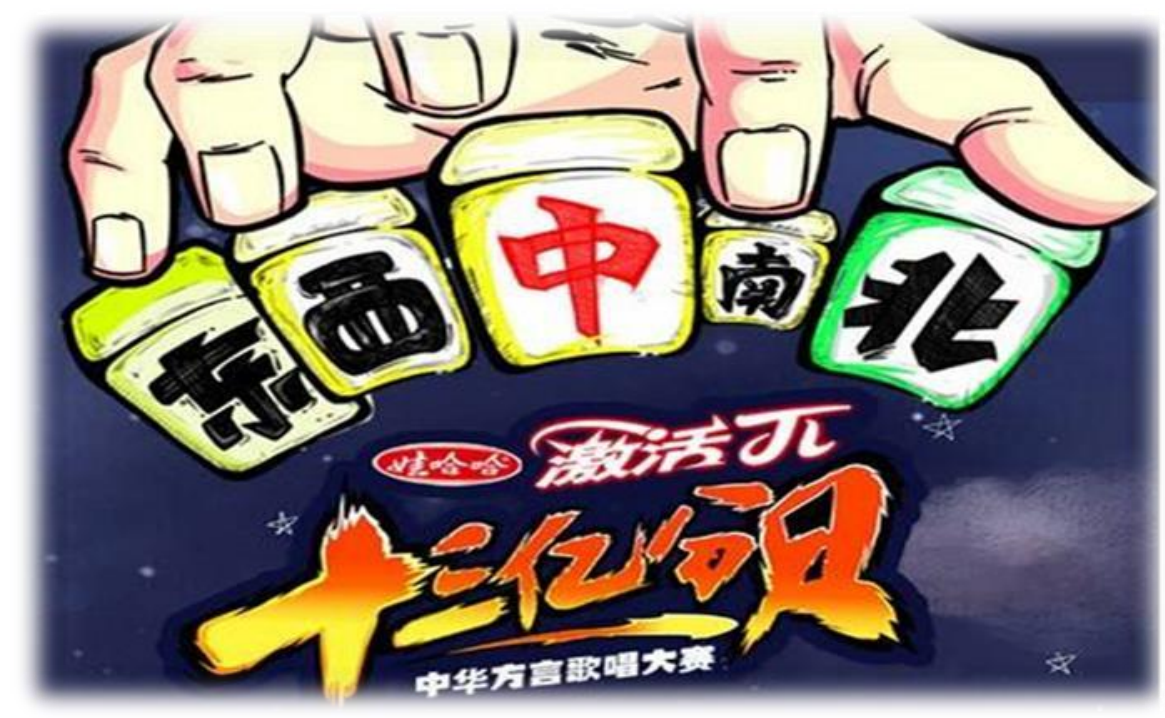

Figure 2. "The 1.3 Billion Decibel" show

\subsection{Data Collection and Analysis}

For Data-collecting procedure, I have done an online ethnography. I spent about 24 hours in total watching the show. The data is collected from the dialogues, reports, singers" introduction, songs, and images from the "1.3 Billion Decibel" show, and the calculation of the language distributions based on the number and frequency of the representations in songs and geography. What's more, content analysis (Shardakova \& Pavlenko, 2004) is adopted to explore the representations of different Fangyan.

\section{Findings}

In this section, participants' language practices in and outside of show are examined with a particular focus on how the skill of speaking Fangyan influences their lives and the representation of fangyan embedded in the Putonghua promotion policy.

\subsection{The Commodification of Fangyan Through Social Media Promotion}

Firstly, Fangyan is capitalized as an index of authenticity and authority. This is a song written by the singer Wang Jianfang from Xi'an, called Chang'anye. Singing this song in Xi'an Fangyan, it can convey a sense of authenticity given that the content is about Xi' an and history, and Xi'an is one of the ancient cities in China. Furthermore, by quoting some iconic figures and items such as "Li Bai", "resting station every $5 \mathrm{~km}$ " (十里长 亭), “thousands of years” (千年), “incarnation” (轮回), “preexistence” (前生), Fangyan can carry the meaning to claim the authority of the story described in the song. 


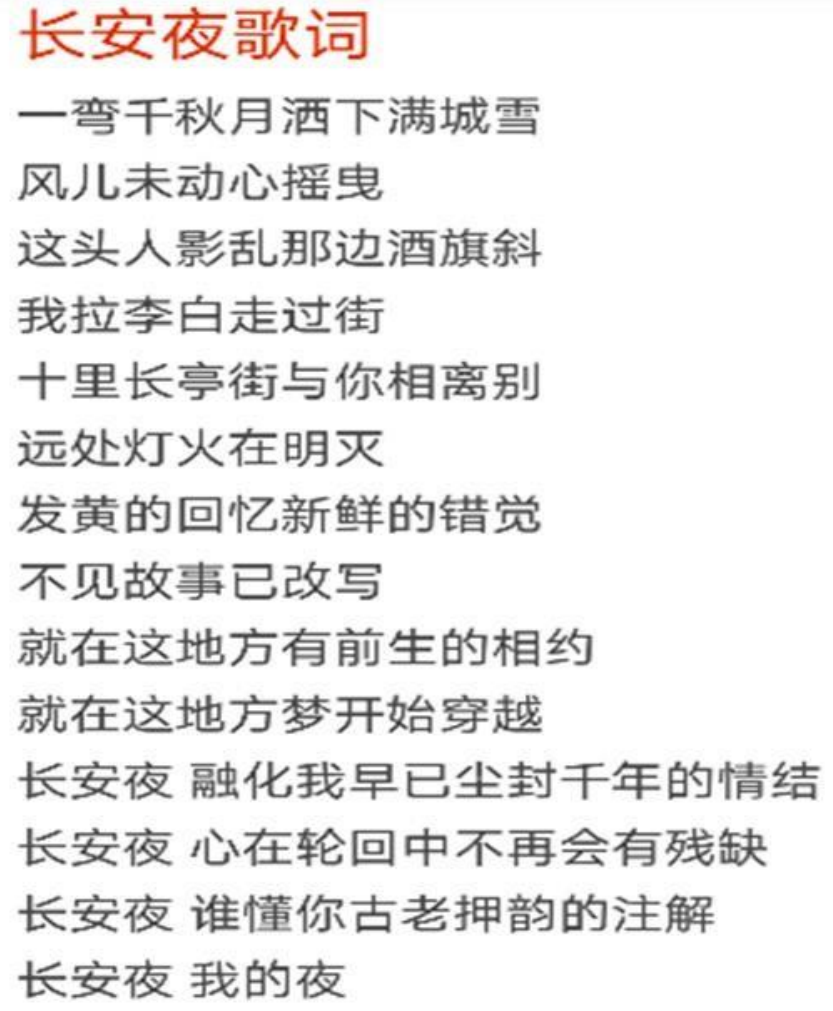

Figure 3. The lyrics of Chang'anye

Secondly, Fangyan is capitalized as a source of knowledge dissemination. In the show, there are two singers both from Xin Jiang. However, one speaks Xinjiang Fangyan (a kind of Han Fangyan), while another speaks Uyghur. From our stereotype, the people from the Xinjiang Uygur Autonomous Region must speak their ethnic minority language, Uyghur. This example disseminates the knowledge that not all people live in the ethnic minority region must be able to speak the local ethnic minority languages.

What's more, Fangyan is capitalized as a commodified capital. Because of Fangyan, the show gets a lot of attention ("huge Traffic"), which means economic profit. By 2018, 1.3 Billion Decibel had been streamed 300 million times, and nearly 3 million people had live-streamed it online. The main topic of 1.3 Billion Decibel in Weibo and the total volume of each sub-topics about 1.3 Billion Decibel exceeded billion, and it topped the list of hot topics for many times $(\mathrm{Gu}, 2018)$. Fangyan is the selling point of the show. Besides, the huge traffic also shows people's consumption needs of Fangyan. For its great achievement, the show won the "Annual Top Ten Brand Influential Online Show". For individual, Fangyan work as a "stepstone" to get education opportunity. Zhou Yulou, born after 1995 in Yueyang, China, was admitted to the Department of Linguistics at Stanford University by "the stepping stone", that is, his love and experience of studying Fangyan. He said "there are only 3 Linguistics majors in the class of 2016 at Stanford University, and I am the only one from China. Many scholars and professors are interested in Chinese Fangyan". It is Fangyan give him the opportunity!

\subsection{The Tensions in the Process of Commodifying Fangyan}

According to Heller (2010)'s theory, during the process of the commodification of language, "there are tensions between standardization and variability, which represents a gap, or troubled space of contradiction, between established nationalist". In the study, three tensions are found: tension between the legitimate language, Putonghua, and the other language varieties, Fangyans, contradiction between the majority Han Fangyan with more people speaking, and the ethnic minority language with more language varieties, and the gaps between goal and real practice in the process of commodifying of Fangyan through social media promotion.

Fangyans are still the secondary choice compared with the legitimate language, Putonghua. In Figure.4, the singer is singing a Uyghur rap. The Uyghur, as an ethnic language in China, has its own characters. However, the characters are not visible. Instead, Hanzi (the written form of Putonghua) is used to show the meaning of the Uyghur song. It gives the sense that Hanzi is with higher prestige, while other Fangyans are invisible. In such a Fangyan promotion show, it should focus on the Fangyan. However, it cannot cast off the dominant place of 
Hanzi and Putonghua.

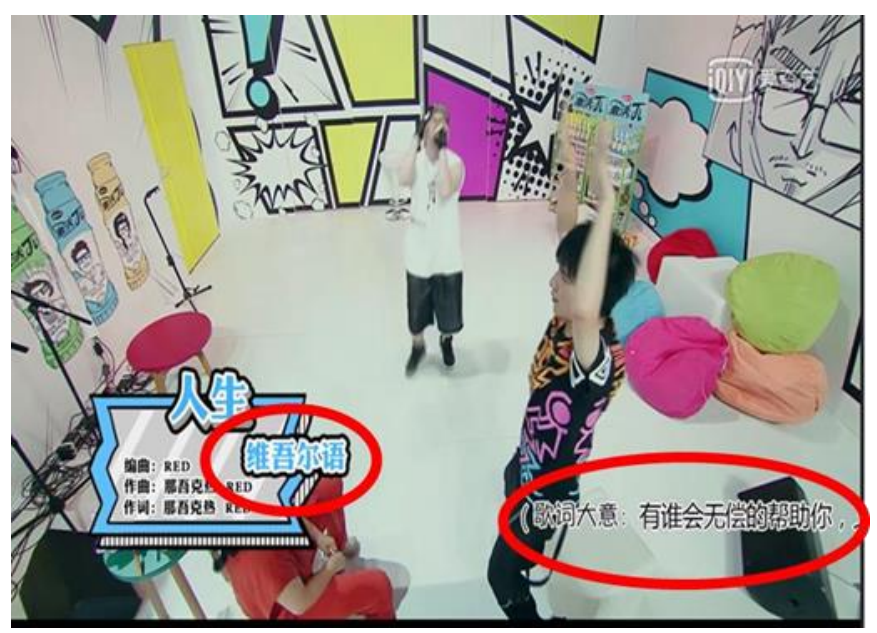

Figure 4. Uyghur song with Han Caption

In the show, Han-Fangyans are placed in a dominant place, while ethnic minority languages are simplified and marginalized. Table.1 is the Geographical distribution of singers in "1.3 Billion Decibel". It shows that the singers mainly from Han regions, such as Hunan, 3 Northeast cities of China: Heilongjiang, Jilin, Liaoning. From the Table 2, it shows that Han ethnic Fangyan dominated whereas other minorities under represented. 95.2\% songs are using Han Fangyan, but the ethnic minority languages songs only take the proportion of $4.8 \%$. The ethnic minority languages are marginalized. As for the ethnic minority languages, there are only 3 kind of ethnic minority language: Yi, Uyghur, Tibetan, while there are 55 national minorities in China. The ethnic minority languages are simplified. Besides, it is found that there are many minority groups and languages in Yunnan, while only Kunming Fangyan appears in the show (In episode16.07.29).

Table 1. Geographical distribution of singers in "1.3 Billion Decibel"

\begin{tabular}{cccc}
\hline Place & Number & Place & Number \\
\hline Hunan & 7 & Tianjin & 2 \\
3 northeast provinces & 6 & Mixed areas & 2 groups \\
Hubei & 5 & Hebei & 1 \\
Guangdong & 5 & Shanxi & 1 \\
Sichuan & 5 & Shandong & 1 \\
Shaanxi & 5 & Guizhou & 1 \\
Jiangsu & 4 & Yunnan & 1 (Kunming fangyan) \\
Fujian & 4 & Taiwan & 1 \\
Beijing & 4 & Guangxi & 1 \\
Chongqing & 3 & Tibet & 1 \\
Xinjiang & 3 & Ningxia & 1 \\
Henan & 2 & & \\
\hline
\end{tabular}

Table 2. The language distribution of songs in "1.3 Billion Decibel"

\begin{tabular}{cccc}
\hline Languages of songs & Han Fangyan & Ethnic minority language & total \\
\hline Number of songs & $119(95.2 \%)$ & $6(4.8 \%)$ & $125(100 \%)$ \\
\hline
\end{tabular}

Besides, there exist some gaps between goal and real practice. The show aims to give people more show for all the people, while it has some restrictions. On the one hand, it has the language restriction. For the singer, he/she must be able to use Putonghua if he/she want to take part in the show, because it is the Putonghua- medium show 
(using Putonghua to give order and communicate with each other). On the other hand, it has set some age restriction. For the audience, they must have the ability to use social medium, and non-netizens are mainly concentrated in the elderly (Over 60 years old) (Mo, 2021). Therefore, the old, who might have the better competence of speaking idiomatic Fangyan cannot participate in the show.

\section{Discussion}

Previous studies conducted in minority languages demonstrate that the increasing visibility and audibility of fangyan has been observed. $\mathrm{Li}$ (2020) also pointed out that Fangyan could be capitalized as an index of authority and authenticity, as a source of success and knowledge dissemination, and as a commodified capital. Agreeing with the previous study, the commodification of Fangyan through social media promotion in the study also take the way to analyze the data. However, according to Monica Heller (2010), the tensions should not be ignored in the commodification of language. This study not only examines the capital of Fangyan, but also pays attention to the tensions behind the commodifying.

\section{Conclusion}

Fangyan is commodified and revitalized under the promotion of social media. Access to learning about linguistic diversity and online resources provide with a third space to reconstruct the connection with Fangyan in the tensions between power and social justice. However, there still exists contradiction between language as authority and language as anonymity, and the idea of multicultural prosperity should not be exaggerated. Finally, such a revitalization of Fangyan or revitalization of linguistic diversity is conditional and nation-based rather than seeing diversity as social realities.

Linguistic diversity is a social reality that was, is and will be in existence. The choices of language/form are also a form of social practices and are embedded in language ideologies and political economy of language. Finally, I hope this study can give reference for the government to manage the language resources that linguistic diversity needs to be placed as the center of sustainable inquiry for social participation.

\section{References}

Cameron, D. (2005). Communication and commodification: Global economic change in sociolinguistic perspective. In G. Erreygers (Ed.), Language, Communication and the Economy (pp. 9-23). Amsterdam: John Benjamins. https://doi.org/10.1075/dapsac.16.04cam

Cao, Z. Y. (2017). Cross the gap: Finding the most effective way for language protection. Language Application, 2, 2-8. https://doi.org/10.16499/j.cnki.1003-5397.2017.02.001

Coupland, N. (2003). Sociolinguistic Authenticities. J7. Sociolinguist, 7(3), 417-31. https://doi.org/10.1111/1467-9481.00233

Dong, J. (2014). Linguistic Identity from the Perspective of Ethnography: Two Cases of Children of Beijing Migrant Workers. Linguistic Research, (1), 155-164.

Fang, Y. (2015). Research on Dialect Dissemination and Identity Recognition of Migrant Workers in the Process of Urbanization. News University, (2), 88-91+143.

Gu, H. Z. (2018). The way to success of the dialect network variety show "1.3 billion decibels"-From the perspective of media niche theory. Journal of Southeast Communication, (3), 131-133.

Heller, M. (2010). The Commodification of Language. Annual Review of Anthropology, 39, 101-114. https://doi.org/10.1146/annurev.anthro.012809.104951

Li, J. (2020). Coronavirus Meets Linguistic Diversity. Retrieved from https://www.languageonthemove.com/coronavirus-meets-linguistic-diversity/

Li, J., \& Lv, L. (2020). Power to Fanyan! Retrieved from https://www.languageonthemove.com/power-to-fanyan/

Li, J., \& Lv, Y. (2020). Minority Languages on the Rise? Retrieved from https://www.languageonthemove.com/minority-languages-on-the-rise/

Mo, K. W. (2021, February 8). The number and structure of Chinese Internet users highlight three major issues. International Finance News, 3.

Shardakova, M., \& Pavlenko, A. (2004). Identity options in Russian textbooks. Journal of Language, Identity and Education, 53, 25-46. https://doi.org/10.1207/s15327701jlie0301_2

Spitulnik, D. (1996). The Social Circulation of Media Discourse and the Mediation of Communities. Journal of 
Linguistic Anthropology, 6(2), 161-187. https://doi.org/10.1525/jlin.1996.6.2.161

Spitulnik, D. (1999). Key Concepts in Linguistic Anthropology. Journal of Linguistic Anthropology, 9(1/2), 148-151. https://doi.org/10.1525/jlin.1999.9.1-2.148

Tian, L. X. (2015). The Origin and Significance of the Chinese Language Resources Protection Project. Language Application, 4, 2-9.

Urciuoli, B. (2001). Review of Good to Talk? Living and Working in a Communication Culture, by D. Cameron. Language in Society, 30(2), 272-275. https://doi.org/10.1017/S0047404501232059

Urla, J. (1995). Outlaw Language: Creating Alternative Public Sphere in Basque Free Radio. Pragmatics, 5(2), 245-261. https://doi.org/10.1075/prag.5.2.09url

\section{Copyrights}

Copyright for this article is retained by the author(s), with first publication rights granted to the journal.

This is an open-access article distributed under the terms and conditions of the Creative Commons Attribution license (http://creativecommons.org/licenses/by/4.0/). 